\title{
DIFFERENTIATION BASES FOR SOBOLEV FUNCTIONS ON METRIC SPACES
}

\author{
Petteri Harjulehto and Juha Kinnunen
}

Abstract

We study Lebesgue points for Sobolev functions over other collections of sets than balls. Our main result gives several conditions for a differentiation basis, which characterize the existence of Lebesgue points outside a set of capacity zero.

\section{Introduction}

By the classical Lebesgue differentiation theorem almost every point is a Lebesgue point for a locally integrable function $f: \mathbf{R}^{n} \rightarrow[-\infty, \infty]$. In particular, this implies that

$$
\lim _{r \rightarrow 0} \frac{1}{|B(x, r)|} \int_{B(x, r)} f(y) d y=f(x)
$$

for almost every $x \in \mathbf{R}^{n}$, where $|\cdot|$ denotes the Lebesgue measure and $B(x, r)$ is a ball with the center $x$ and the radius $r>0$. It is a very interesting question to ask whether (1.1) holds for other collections of sets than balls. This question has been studied extensively in [dG1] and $[\mathbf{d G 2}$, see also Chapter 10 of $[\mathbf{S t}]$. It turns out that the existence of the Lebesgue points is equivalent to certain estimates for maximal functions and derivatives of the integrals as well as to some covering properties.

The objective of this note is to study similar questions for functions which are more regular. Indeed, we are interested in functions, which belong to a first order Sobolev space. It is known that (1.1) holds for a Sobolev function outside a set of capacity zero, see $[\mathbf{E G}]$. We are interested in limits of integral averages over other collections of sets than balls. Our main theorem gives several equivalent conditions for a differentiation basis, which characterize the existence of Lebesgue points outside a set of capacity zero. An interesting feature of the result is 
that a qualitative result, as the existence of the Lebesgue points, implies quantitative estimates. Another interesting fact is that certain capacitary weak type estimates for maximal functions or derivatives of the integrals imply the existence of the Lebesgue points. These phenomena are visible already in $[\mathbf{d G} \mathbf{1}]$ and $[\mathbf{d G} \mathbf{2}]$. In fact, our proofs are modifications of the corresponding arguments for integrable functions, but the proofs for Sobolev functions are somewhat more subtle.

To emphasize the fact that our proof is based on a general principle we state and prove our main result in the context of metric measure spaces. Recently there has been some interest in defining the first order Sobolev spaces on a metric measure space, see $[\mathbf{C}],[\mathbf{F K S}],[\mathbf{H a}],[\mathbf{H K M}]$, $[\mathbf{H e K o}]$ and $[\mathbf{S h}]$ suitable modifications our argument applies to any of these approaches. For simplicity, we have chosen the definition of Sobolev spaces on a metric measure space due to Hajłasz [Ha]. A general outline of the theory and further references can be found in [HaKo2]. The Lebesgue theorem with respect to balls for Sobolev functions on metric spaces has been studied in $[\mathbf{K L}]$. In the final section we briefly comment on the Euclidean case.

\section{Preliminaries}

In this section we recall the definition due to Hajłasz [Ha] of a first order Sobolev space on an arbitrary metric measure space. Let $(X, d)$ be a metric space and let $\mu$ be a non-negative Borel regular outer measure on $X$. In the following, we keep the metric measure space $(X, d, \mu)$ fixed, and for short, we denote it by $X$. The Lebesgue space $L^{p}(X)$ with $1 \leq p<\infty$ is the Banach space of all $\mu$-a.e. defined $\mu$-measurable functions $f: X \rightarrow[-\infty, \infty]$ with the norm

$$
\|f\|_{L^{p}(X)}=\left(\int_{X}|f|^{p} d \mu\right)^{1 / p} .
$$

The space $L^{\infty}(X)$ consists of essentially bounded functions.

Let $1<p \leq \infty$ and suppose that $u \in L^{p}(X)$. We denote by $D(u)$ the set of all $\mu$-measurable functions $g: X \rightarrow[0, \infty]$ such that

$$
|u(x)-u(y)| \leq d(x, y)(g(x)+g(y))
$$

for every $x, y \in X \backslash N, x \neq y$, with $\mu(N)=0$. In the metric setting, instead of having the gradient, we have a set $D(u)$ of maximal gradients of $u$. A function $u \in L^{p}(X)$ belongs to the Sobolev space $M^{1, p}(X)$ if

$$
D(u) \cap L^{p}(X) \neq \emptyset .
$$


The Sobolev space $M^{1, p}(X)$ is equipped with the norm

$$
\|u\|_{M^{1, p}(X)}=\left(\|u\|_{L^{p}(X)}^{p}+\|u\|_{L^{1, p}(X)}^{p}\right)^{1 / p},
$$

where

$$
\|u\|_{L^{1, p}(X)}=\inf \left\{\|g\|_{L^{p}(X)}: g \in D(u) \cap L^{p}(X)\right\} .
$$

We recall some basic properties of the Sobolev space $M^{1, p}(X)$. If $u \in M^{1, p}(X)$ and $g \in D(u) \cap L^{p}(X)$, then the Poincaré inequality

$$
f_{B(x, r)}\left|u-u_{B(x, r)}\right| d \mu \leq 2 r f_{B(x, r)} g d \mu
$$

holds for every $x \in X$ and $r>0$. Here we use the standard notation

$$
f_{B(x, r)}=f_{B(x, r)} f d \mu=\frac{1}{\mu(B(x, r))} \int_{B(x, r)} f d \mu
$$

and $B(x, r)$ denotes the open ball with the center $x$ and the radius $r>0$. The Poincaré inequality is easily proved by integrating the pointwise inequality (2.1) twice over the ball.

It follows immediately from the definition that if $u \in M^{1, p}(X)$ then $|u| \in M^{1, p}(X)$ with

$$
\||u|\|_{M^{1, p}(X)} \leq\|u\|_{M^{1, p}(X)} .
$$

If $X=\mathbf{R}^{n}$ with the Euclidean metric and the Lebesgue measure, then

$$
M^{1, p}\left(\mathbf{R}^{n}\right)=W^{1, p}\left(\mathbf{R}^{n}\right), \quad 1<p \leq \infty .
$$

Moreover, the norms are comparable (see $[\mathbf{H a}]$ ). Here $W^{1, p}\left(\mathbf{R}^{n}\right)$ is the first order Sobolev space of those functions in $L^{p}\left(\mathbf{R}^{n}\right)$ whose first distributional derivatives belong to $L^{p}\left(\mathbf{R}^{n}\right)$ with the norm

$$
\|u\|_{W^{1, p}\left(\mathbf{R}^{n}\right)}=\|u\|_{L^{p}\left(\mathbf{R}^{n}\right)}+\|\nabla u\|_{L^{p}\left(\mathbf{R}^{n}\right)} .
$$

Indeed, if $u \in W^{1, p}\left(\mathbf{R}^{n}\right)$, then we have the pointwise inequality

$$
|u(x)-u(y)| \leq c|x-y|(M|\nabla u|(x)+M|\nabla u|(y))
$$

for every $x, y \in \mathbf{R}^{n} \backslash N$ with $|N|=0$. Here $M|\nabla u|$ is the HardyLittlewood maximal function of $|\nabla u|$. The maximal operator is bounded in $L^{p}\left(\mathbf{R}^{n}\right)$ when $1<p \leq \infty$. This shows that $M|\nabla u| \in D(u) \cap L^{p}\left(\mathbf{R}^{n}\right)$ and hence $W^{1, p}\left(\mathbf{R}^{n}\right) \subset M^{1, p}\left(\mathbf{R}^{n}\right)$. The reverse inclusion follows from the characterization of $W^{1, p}\left(\mathbf{R}^{n}\right)$ with the integrated difference quotients, see 7.11 of $[\mathbf{G T}]$. Since the maximal operator is not bounded in $L^{1}\left(\mathbf{R}^{n}\right)$ the case $p=1$ is excluded in the definition. This also suggests that $g \in D(u)$ corresponds to the maximal function of the gradient of $u$ rather than the gradient. 
There is a natural capacity in the Sobolev space. For $1<p<\infty$, the Sobolev p-capacity of the set $E \subset X$ is the number

$$
C_{p}(E)=\inf \left\{\|u\|_{M^{1, p}(X)}^{p}: u \in \mathcal{A}(E)\right\},
$$

where

$$
\mathcal{A}(E)=\left\{u \in M^{1, p}(X): u \geq 1 \text { on an open neighbourhood of } E\right\} .
$$

If $\mathcal{A}(E)=\emptyset$, we set $C_{p}(E)=\infty$. The Sobolev capacity is a monotone and countably subadditive set function, see Theorem 3.2 in $[\mathbf{K M}]$. It is easy to see [KM, Remark 3.3] that the Sobolev capacity is an outer capacity, which means that

$$
C_{p}(E)=\inf \left\{C_{p}(O): O \supset E, O \text { open }\right\} .
$$

The capacity measures the exceptional sets for Sobolev functions. To be more precise, a function $u: X \rightarrow[-\infty, \infty]$ is p-quasi continuous in $X$ if for every $\varepsilon>0$ there is a set $E$ such that $C_{p}(E)<\varepsilon$ and the restriction of $u$ to $X \backslash E$ is continuous. By outer regularity, we may assume that $E$ is open. Functions in $M^{1, p}(X)$ are defined only up to a set of measure zero, but the following result [KM, Corollary 3.7] shows that Sobolev functions are defined outside a set of capacity zero.

Theorem 2.3. For each $u \in M^{1, p}(X)$ there is a p-quasi continuous function $u^{*} \in M^{1, p}(X)$ such that $u=u^{*} \mu$-a.e. in $X$.

Moreover, the $p$-quasi continuous representative is unique in the sense that if two $p$-quasi continuous functions coincide $\mu$-almost everywhere, then they actually coincide outside a set of $p$-capacity zero. For a proof of this we refer to [Kil]. We say that a property holds p-quasi everywhere if it holds outside a set of $p$-capacity zero.

There is a useful characterization of the capacity in terms of quasi continuous functions. Indeed

$$
C_{p}(E)=\inf \left\{\|u\|_{M^{1, p}(X)}^{p}: u \in \mathcal{Q A}(E)\right\},
$$

where

$$
\begin{aligned}
\mathcal{Q A}(E)=\left\{u \in M^{1, p}(X): u \text { is } p\right. \text {-quasi continuous and } \\
\qquad u \geq 1 p \text {-quasi everywhere on } E\} .
\end{aligned}
$$

This will be a crucial fact for us later. For the proof we refer to Theorem 3.4 in [KKM].

Following $[\mathbf{d G} \mathbf{1}]$ and $[\mathbf{d G} \mathbf{2}]$ we say that a differentiation basis in $X$ is a collection

$$
\mathcal{B}=\bigcup\{\mathcal{B}(x): x \in X\}
$$


of bounded measurable sets with positive measure such that for every $x \in X$ there is a subfamily $\mathcal{B}(x)$ of sets of $\mathcal{B}$ so that $x$ is contained in every $B \in \mathcal{B}(x)$ and $\mathcal{B}(x)$ contains sets of arbitrarily small diameter.

Let $f \in L_{\mathrm{loc}}^{1}(X)$. We define the upper derivative of $f$ with respect to the differentiation basis $\mathcal{B}$ as $\bar{D} f: X \rightarrow[0, \infty]$ with

$$
\bar{D} f(x)=\limsup _{\delta_{x}(B) \rightarrow 0} f_{B} f(y) d \mu(y),
$$

where $\delta_{x}(B)=\operatorname{diam}(B)$ and $B \in \mathcal{B}(x)$. Recall that

$$
f_{B(x, r)} f(y) d \mu(y)=\frac{1}{\mu(B(x, r))} \int_{B(x, r)} f(y) d \mu(y) .
$$

The lower derivative $\underline{D} f(x)$ can be defined in the same way by replacing the limes superior by limes inferior. If $\underline{D} f(x)=\bar{D} f(x)$, we say that the derivative

$$
D f(x)=\lim _{\delta_{x}(B) \rightarrow 0} f_{B} f(y) d \mu(y)
$$

exists at $x$. Observe, that if $f$ is continuous at $x \in X$, then the derivative exists and $f(x)=D f(x)$.

Definition 2.7. Suppose that $1<p<\infty$. We say that a differentiation basis $\mathcal{B}$ differentiates the Sobolev space $M^{1, p}(X)$ if for every $u \in M^{1, p}(X)$ the derivative $D u(x)$ exists $p$-quasi everywhere in $X$ and

$$
D u(x)=u^{*}(x)
$$

$p$-quasi everywhere in $X$, where $u^{*}$ is a $p$-quasi continuous representative of $u$.

Remarks 2.8. (1) By Theorem 1.2 it suffices to check that

$$
D u^{*}(x)=u^{*}(x)
$$

$p$-quasi everywhere in $X$, where $u^{*}$ is a $p$-quasi continuous representative of $u$.

(2) If $\mathcal{B}$ differentiates $M^{1, p}(X)$, then for every $u \in M^{1, p}(X)$, we have

$$
\lim _{\delta_{x}(B) \rightarrow 0} f_{B}\left|u(y)-u^{*}(x)\right| d \mu(y)=0
$$

for $p$-quasi every $x \in X$ where $u^{*}$ is a $p$-quasi continuous representative of $u$. In other words, $p$-quasi every point is a Lebesgue point of $u$.

By subadditivity it is enough to verify the claim in a ball $B(z, r)$, where $z \in X$ and $r>0$. Let $\phi$ be a Lipschitz continuous cut-off function such that $0 \leq \phi \leq 1, \phi=1$ in $B(z, r)$ and $\phi=0$ in $X \backslash B(z, 2 r)$. Let $q_{k}$, 
$k=1,2, \ldots$, be an enumeration of rational numbers. For every $q_{k}$ the function $\left|\phi\left(u^{*}-q_{k}\right)\right|$ is a $p$-quasi continuous representative of $\left|\phi\left(u-q_{k}\right)\right|$. By Definition 2.7 there is a set $E_{k} \subset X$ of $p$-capacity zero such that

$$
\lim _{\delta_{x}(B) \rightarrow 0} f_{B}\left|\phi(y)\left(u(y)-q_{k}\right)\right| d y=\left|\phi(x)\left(u^{*}(x)-q_{k}\right)\right|
$$

for all $x \in X \backslash E_{k}$. Let $E=\bigcup_{k=1}^{\infty} E_{k}$. Then $C_{p}(E)=0$ and (2.10) holds for every $x \in X \backslash E$. Let

$$
F=\left\{x \in X:\left|u^{*}(x)\right|=\infty\right\} .
$$

By (2.4) we may use $u^{*}$ as a test function and we obtain

$$
\mathrm{C}_{p}(F) \leq \mathrm{C}_{p}\left(\left\{x \in X:\left|u^{*}(x)\right|>\lambda\right\}\right) \leq \lambda^{-p}\|u\|_{M^{1, p}(X)}^{p} .
$$

Letting $\lambda$ tend to infinity we arrive at $\mathrm{C}_{p}(F)=0$. Let $x \in X \backslash(E \cup F)$ and let $\varepsilon>0$. Let $q_{k}$ be a rational number such that $\left|u^{*}(x)-q_{k}\right|<\varepsilon / 2$. Then

$$
\begin{aligned}
\limsup _{\delta_{x}(B) \rightarrow 0} f_{B} & \left|\phi(y)\left(u(y)-u^{*}(x)\right)\right| d \mu(y) \\
\leq & \limsup _{\delta_{x}(B) \rightarrow 0} f_{B}\left|\phi(y)\left(u(y)-q_{k}\right)\right| d \mu(y) \\
& +\limsup _{\delta_{x}(B) \rightarrow 0} f_{B}\left|\phi(y)\left(q_{k}-u^{*}(x)\right)\right| d \mu(y) \\
= & 2\left|\phi(x)\left(u^{*}(x)-q_{k}\right)\right|<\varepsilon .
\end{aligned}
$$

Since $\varepsilon>0$ is arbitrary, we obtain (2.9) for every $x \in B(z, r) \backslash(E \cup F)$.

The following theorem is our main result.

Theorem 2.11. The following claims are equivalent:

(i) $\mathcal{B}$ differentiates $M^{1, p}(X)$.

(ii) There is a constant $c$ such that

$$
C_{p}(\{x \in X: \bar{D}|u|(x)>\lambda\}) \leq c \lambda^{-p}\|u\|_{M^{1, p}(X)}^{p}
$$

for every $\lambda>0$ and for every $u \in M^{1, p}(X)$.

(iii) Let $u_{i} \in M^{1, p}(X), i=1,2, \ldots$, and suppose that $\left\|u_{i}\right\|_{M^{1, p}(X)} \rightarrow 0$ as $i \rightarrow \infty$. Then for each $\lambda>0$ we have

$$
C_{p}\left(\left\{x \in X: \bar{D}\left|u_{i}\right|(x)>\lambda\right\}\right) \rightarrow 0
$$

as $i \rightarrow \infty$.

(iv) For every $u \in M^{1, p}(X)$ we have

$$
C_{p}(\{x \in X: \bar{D}|u|(x)>\lambda\}) \rightarrow 0
$$

as $\lambda \rightarrow \infty$. 
Observe, that the claim (ii) is quantitative but the claims (i), (iii) and (iv) are qualitative.

Remarks 2.12. (1) Recall that $\mu$ is a doubling measure if there is a constant $c_{\mu} \geq 1$ so that

$$
\mu(B(x, 2 r)) \leq c_{\mu} \mu(B(x, r))
$$

for every open ball $B(x, r)$ in $X$. An iteration of the doubling property implies, that if $B(y, R)$ is a ball in $X, z \in B(y, R)$ and $0<r \leq R<\infty$, then

$$
\frac{\mu(B(z, r))}{\mu(B(y, R))} \geq c\left(\frac{r}{R}\right)^{Q}
$$

for some $c=c\left(c_{\mu}\right)$ and $Q=\log c_{\mu} / \log 2$. The exponent $Q$ serves as a counterpart of dimension related to the measure and, for example, in $\mathbf{R}^{n}$ with the Lebesgue measure $Q$ is equal to the dimension $n$.

A result in [HaKo1] (see also Theorem 5.1 in [HaKo2]) shows that if $\mu$ is doubling, then the Poincaré inequality (2.2) implies a SobolevPoincaré inequality. More precisely, if $1<p<Q$ then for every $\kappa$ with $1 \leq \kappa<Q /(Q-p)$ there is $c=c\left(p, \kappa, c_{\mu}\right)>0$ depending on such that

$$
\left(f_{B(z, r)}\left|u-u_{B(z, r)}\right|^{\kappa p} d \mu\right)^{1 /(\kappa p)} \leq c r\left(f_{B(z, 5 r)} g^{p} d \mu\right)^{1 / p}
$$

for every $g \in D(u) \cap L^{p}(X)$. If $p>Q$, then

$$
|u(x)-u(y)| \leq c r^{Q / p} d(x, y)^{1-Q / p}\left(f_{B(z, 5 r)} g^{p} d \mu\right)^{1 / p}
$$

for every $x, y \in B(z, r) \backslash N$ with $\mu(N)=0$ and $g \in D(u) \cap L^{p}(X)$. In particular, this implies that, after a redefinition on a set of measure zero, functions in $M^{1, p}(X)$ with $p>Q$ are Hölder continuous on bounded subsets of $X$. In the borderline case $p=Q$ there is an exponential estimate, but we do not need it here. This implies, in particular, that if the measure is doubling, then every differentiation basis differentiates $M^{1, p}(X)$ when $p>Q$.

(2) A standard way to verify the conditions (ii), (iii) or (iv) for a differentiation basis $\mathcal{B}$ is to consider the corresponding Hardy-Littlewood maximal function. The maximal function related to the differentiation basis $\mathcal{B}$ of a $f \in L_{\text {loc }}^{1}(X)$ is defined as

$$
M f(x)=\sup _{B \in \mathcal{B}(x)} f_{B}|f(y)| d \mu(y) .
$$


It is clear that

$$
\bar{D}|f|(x) \leq M f(x)
$$

for every $x \in X$ so that, for instance, if we are able to prove the weak type estimate

$$
C_{p}(\{x \in X: M u(x)>\lambda\}) \leq c \lambda^{-p}\|u\|_{M^{1, p}(X)}^{p}
$$

for every $\lambda>0$, then we obtain (ii). For the differentiation basis consisting of balls this estimate was proved in $[\mathbf{K L}]$. In particular, this implies that the basis consisting of balls differentiates $M^{1, p}(X)$ when $1<p<\infty$ and the measure is doubling.

\section{Proofs of the equivalencies}

The proofs are rather straightforward modifications of the corresponding results for the Lebesgue measure in $[\mathbf{d G} \mathbf{1}]$ and $[\mathbf{d G} \mathbf{2}]$. However, the lack of measurable sets for the capacity has the effect that proofs are somewhat subtle.

Lemma 3.1. The conditions (i) and (ii) are equivalent.

Proof: First we show that the condition (i) implies (ii). Let $u \in M^{1, p}(X)$. Since $\mathcal{B}$ differentiates $M^{1, p}(X)$ and $|u| \in M^{1, p}(X)$, we have

$$
\bar{D}|u|(x)=|u|^{*}(x)
$$

$p$-quasi everywhere in $X$ for every $p$-quasi continuous representative $|u|^{*}$ of $|u|$. In particular, this implies that $\bar{D}|u|$ is $p$-quasi continuous. By (2.4) we may use $\lambda^{-1} \bar{D}|u|, \lambda>0$, as a test function and we obtain

$$
\begin{aligned}
C_{p}(\{x \in X: \bar{D}|u|(x)>\lambda\}) & \leq \lambda^{-p}\left\||u|^{*}\right\|_{M^{1, p}(X)}^{p} \\
& =\lambda^{-p}\|\mid u\|_{M^{1, p}(X)}^{p} \leq \lambda^{-p}\|u\|_{M^{1, p}(X)}^{p} .
\end{aligned}
$$

This is the desired estimate.

Then we show that (ii) implies (i). Suppose that $u \in M^{1, p}(X)$ and let $u^{*}$ be a $p$-quasi continuous representative of $u$. By Remark 2.8 (1) it suffices to prove that

$$
D u^{*}(x)=u^{*}(x)
$$

for $p$-quasi every $x \in X$. Since continuous functions are dense in $M^{1, p}(X)$ there is a continuous function $v \in M^{1, p}(X)$ and a $p$-quasi continuous function $w \in M^{1, p}(X)$ such that $u^{*}=v+w$ and that $\|w\|_{M^{1, p}(X)}$ is as small as we please. From this we conclude that

$$
\bar{D}\left|u^{*}-u^{*}(x)\right|(x) \leq \bar{D}|w-w(x)|(x) \leq \bar{D}|w|(x)+|w(x)|
$$


for every $x \in X$ and consequently

$$
\begin{aligned}
C_{p}(\{x & \left.\left.\in X: \bar{D}\left|u^{*}-u^{*}(x)\right|(x)>\lambda\right\}\right) \\
& \leq C_{p}(\{x \in X: \bar{D}|w|(x)>\lambda / 2\})+C_{p}(\{x \in X:|w(x)|>\lambda / 2\}) .
\end{aligned}
$$

The assumption (ii) implies that

$$
C_{p}(\{x \in X: \bar{D}|w|(x)>\lambda / 2\}) \leq c \lambda^{-p}\||w|\|_{M^{1, p}(X)}^{p} \leq c \lambda^{-p}\|w\|_{M^{1, p}(X)}^{p} .
$$

By (2.4) we may use $w$ as a test function and we obtain

$$
C_{p}(\{x \in X:|w(x)|>\lambda / 2\}) \leq 2^{p} \lambda^{-p}\||w|\|_{M^{1, p}(X)}^{p} \leq 2^{p} \lambda^{-p}\|w\|_{M^{1, p}(X)}^{p} .
$$

From these estimates we conclude that

$$
C_{p}\left(\left\{x \in X: \bar{D}\left|u^{*}-u^{*}(x)\right|(x)>\lambda\right\}\right) \leq c \lambda^{-p}\|w\|_{M^{1, p}(X)}^{p},
$$

where $c$ is independent of $u$ and $\|w\|_{M^{1, p}(X)}$ is as small as we please. This shows that

$$
C_{p}\left(\left\{x \in X: \bar{D}\left|u^{*}-u^{*}(x)\right|(x)>\lambda\right\}\right)=0
$$

for every $\lambda>0$. Since

$$
\left\{x \in X: \bar{D}\left|u^{*}-u^{*}(x)\right|(x)>0\right\}=\bigcup_{i=1}^{\infty}\left\{x \in X: \bar{D}\left|u^{*}-u^{*}(x)\right|(x)>1 / i\right\}
$$

subadditivity of the capacity implies that

$$
C_{p}\left(\left\{x \in X: \bar{D}\left|u^{*}-u^{*}(x)\right|(x)>0\right\}\right)=0 .
$$

Therefore $\bar{D}\left|u^{*}-u^{*}(x)\right|(x)=0$ for $p$-quasi every $x \in X$ and since

$$
\left|f_{B} u^{*}(y) d y-u^{*}(x)\right| \leq f_{B}\left|u^{*}(y)-u^{*}(x)\right| d y
$$

for every $B \in \mathcal{B}(x)$ we conclude that $D u^{*}(x)$ exists and $D u^{*}(x)=u^{*}(x)$ for $p$-quasi every $x \in X$. This completes the proof.

Lemma 3.2. The conditions (ii) and (iii) are equivalent.

Proof: Since (ii) clearly implies (iii), it is enough to prove that (iii) implies (ii). Let $u \in M^{1, p}(X)$. Since continuous functions are dense in $M^{1, p}(X)$ there are continuous functions $v_{i} \in M^{1, p}(X), i=1,2, \ldots$, such that

$$
\left\|u-v_{i}\right\|_{M^{1, p}(X)} \rightarrow 0
$$


as $i \rightarrow \infty$. Let $w_{i}=u-v_{i}, i=1,2, \ldots$ Then

$C_{p}(\{x \in X: \bar{D}|u|(x)>\lambda\})$

$$
\begin{aligned}
& =C_{p}\left(\left\{x \in X: \bar{D}\left|v_{i}+w_{i}\right|(x)>\lambda\right\}\right) \\
& \leq C_{p}\left(\left\{x \in X: \bar{D}\left|v_{i}\right|(x)>\lambda / 2\right\}\right)+C_{p}\left(\left\{x \in X: \bar{D}\left|w_{i}\right|(x)>\lambda / 2\right\}\right) \\
& =C_{p}\left(\left\{x \in X:\left|v_{i}(x)\right|>\lambda / 2\right\}\right)+C_{p}\left(\left\{x \in X: \bar{D}\left|w_{i}\right|(x)>\lambda / 2\right\}\right),
\end{aligned}
$$

for every $\lambda>0$. Since each $v_{i} \in M^{1, p}(X)$ is continuous we may use it as a test function for the capacity. This implies that

$$
C_{p}\left(\left\{x \in X:\left|v_{i}(x)\right|>\lambda / 2\right\}\right) \leq 2^{p} \lambda^{-p}\left\|v_{i}\right\|_{M^{1, p}(X)}^{p}
$$

for $i=1,2, \ldots$ Since $\left\|w_{i}\right\|_{M^{1, p}(X)} \rightarrow 0$ as $i \rightarrow \infty$, the condition (iii) implies that

$$
C_{p}\left(\left\{x \in X: \bar{D}\left|w_{i}\right|(x)>\lambda / 2\right\}\right) \rightarrow 0
$$

as $i \rightarrow \infty$. Since $\left\|v_{i}\right\|_{M^{1, p}(X)} \rightarrow\|u\|_{M^{1, p}(X)}$ as $i \rightarrow \infty$, we conclude that

$$
C_{p}(\{x \in X: \bar{D}|u|(x)>\lambda\}) \leq 2^{p} \lambda^{-p}\|u\|_{M^{1, p}(X)}^{p} .
$$

This completes the proof.

Lemma 3.3. The conditions (iii) and (iv) are equivalent.

Proof: We show that (iii) implies (iv). Let $u \in M^{1, p}(X)$. Assume that $\left(\lambda_{i}\right)$ is a sequence with $\lambda_{i} \rightarrow \infty$ as $i \rightarrow \infty$. Since

$$
\left\|\lambda_{i}^{-1}|u|\right\|_{M^{1, p}(X)} \rightarrow 0
$$

as $i \rightarrow \infty$ we obtain by the condition (iii) that

$$
C_{p}\left(\left\{x \in X: \bar{D}|u|(x)>\lambda_{i}\right\}\right)=C_{p}\left(\left\{x \in X: \bar{D}\left(\lambda_{i}^{-1}|u|\right)(x)>1\right\}\right) \rightarrow 0
$$

as $i \rightarrow \infty$.

Then we show that (iv) implies (iii). This proof is based on the uniform boundedness theorem and is a modification of Theorem 1.1.1 of [dG2]. We define the space $Y$ of $\mu$-measurable functions $f$ for which

$$
C_{p}(\{x \in X:|f(x)|>\lambda\}) \rightarrow 0
$$

as $\lambda \rightarrow \infty$. It is not difficult to show that $Y$ is quasi normed space in the sense of Yosida (see p. 31 of $[\mathbf{Y}]$ ) with the quasi norm

$$
d(f)=\inf _{\alpha>0}\left(\alpha+C_{p}(\{x \in X:|f(x)|>\alpha\})\right) .
$$


Let $u_{i} \in M^{1, p}(X), i=1,2, \ldots$, and suppose that $\left\|u_{i}\right\|_{M^{1, p}(X)} \rightarrow 0$ as $i \rightarrow \infty$. By (iv) we have

$$
\bar{D}\left|u_{i}\right| \in Y \quad \text { and } \quad d\left(\bar{D}\left|u_{i}\right|\right)<\infty
$$

for every $i=1,2, \ldots$ The uniform boundedness theorem [Y, Theorem 1, p. 68] implies that $d\left(\bar{D}\left|u_{i}\right|\right) \rightarrow 0$ as $i \rightarrow \infty$. This completes the proof.

\section{Notes on the Euclidean case}

Suppose that the differentiation basis $\mathcal{B}$ differentiates $L^{1}\left(\mathbf{R}^{n}\right)$ in the terminology of [dG1] and [dG2]. This means that for every $f \in L^{1}\left(\mathbf{R}^{n}\right)$ the derivative $D f(x)$ exists almost everywhere in $\mathbf{R}^{n}$ and

$$
D f(x)=f(x)
$$

almost everywhere in $\mathbf{R}^{n}$.

A differentiation basis $\mathcal{B}$ is said to be a Busemann-Feller basis if every $B \in \mathcal{B}$ is open and if $B \in \mathcal{B}$ such that $x \in B$, then $B \in \mathcal{B}(x)$. Moreover, a differentiation basis $\mathcal{B}$ is said to invariant under homotheties if for any $B \in \mathcal{B}$ every set in $\mathbf{R}^{n}$ which is homothetic to $B$ belongs to $\mathcal{B}$. In particular this means that $\mathcal{B}$ is invariant under translations and dilations.

We assume that $\mathcal{B}$ is a Busemann-Feller basis which is invariant under homotheties. Then Theorem 6.4 .10 of [dG2] says that $\mathcal{B}$ differentiates $L^{1}\left(\mathbf{R}^{n}\right)$ if and only if the corresponding maximal function satisfies the weak type estimate

$$
\left|\left\{x \in \mathbf{R}^{n}: M f(x)>\lambda\right\}\right| \leq c \lambda^{-1}\|f\|_{L^{1}\left(\mathbf{R}^{n}\right)}
$$

for every $\lambda>0$ with $c$ independent of $\lambda$. There are also several other equivalent conditions, see Chapter 1 of $[\mathbf{d G} 2]$.

Theorem 4.2. Suppose that $\mathcal{B}$ is a Busemann-Feller basis which is invariant under homotheties and which differentiates $L^{1}\left(\mathbf{R}^{n}\right)$. Then $\mathcal{B}$ differentiates $W^{1, p}\left(\mathbf{R}^{n}\right)$.

Proof: Estimate (4.1) shows that the Hardy-Littlewood maximal operator with respect to $\mathcal{B}$ is of weak type $(1,1)$ and, on the other hand, we have the trivial estimate

$$
\|M f\|_{L^{\infty}\left(\mathbf{R}^{n}\right)} \leq\|f\|_{L^{\infty}\left(\mathbf{R}^{n}\right)} .
$$

Since the maximal operator is sublinear, we may apply the Marcinkiewicz interpolation theorem and we conclude that

$$
\|M f\|_{L^{p}\left(\mathbf{R}^{n}\right)} \leq c\|f\|_{L^{p}\left(\mathbf{R}^{n}\right)}, \quad 1<p \leq \infty,
$$

with a constant $c$ depending only on $p$ and the constant in (4.1). 
Then we use the characterization of Sobolev spaces by integrated difference quotients, see 7.11 of $[\mathbf{G T}]$. Denote $f_{h}(x)=f(x+h)$ for $h \in \mathbf{R}^{n}$. We observe that the maximal operator commutes with translations since $\mathcal{B}$ is invariant under translations. Thus (4.3) gives

$$
\begin{aligned}
\left\|(M u)_{h}-M u\right\|_{L^{p}\left(\mathbf{R}^{n}\right)} & =\left\|M\left(u_{h}\right)-M u\right\|_{L^{p}\left(\mathbf{R}^{n}\right)} \leq\left\|M\left(u_{h}-u\right)\right\|_{L^{p}\left(\mathbf{R}^{n}\right)} \\
& \leq c\left\|u_{h}-u\right\|_{L^{p}\left(\mathbf{R}^{n}\right)} \leq c\|D u\|_{L^{p}\left(\mathbf{R}^{n}\right)}|h| .
\end{aligned}
$$

From this we conclude that the $M u \in W^{1, p}\left(\mathbf{R}^{n}\right)$ and

$$
\|M u\|_{W^{1, p}\left(\mathbf{R}^{n}\right)} \leq c\|u\|_{W^{1, p}\left(\mathbf{R}^{n}\right)},
$$

with a constant $c$ depending only on $p, n$ and the constant in (4.1). Finally we observe that since $\mathcal{B}$ is a Busemann-Feller basis the set $\left\{x \in \mathbf{R}^{n}: M u(x)>\lambda\right\}$ is open for every $\lambda>0$. Thus

$$
C_{p}\left(\left\{x \in \mathbf{R}^{n}: M u(x)>\lambda\right\}\right) \leq\left\|\lambda^{-1} M u\right\|_{W^{1, p}\left(\mathbf{R}^{n}\right)}^{p} \leq c \lambda^{-p}\|u\|_{W^{1, p}\left(\mathbf{R}^{n}\right)}^{p}
$$

for every $\lambda>0$ with a constant $c$ depending only on $p, n$ and the constant in (4.1). This means that the condition (ii) is satisfied and the claim follows.

As a by-product we obtain a generalization of a result in [Kin] to other differentiation basis than the basis consisting of balls.

Corollary 4.5. Suppose that $\mathcal{B}$ is a Busemann-Feller basis which is invariant under homotheties and which differentiates $L^{1}\left(\mathbf{R}^{n}\right)$. Then the maximal function related to the basis $\mathcal{B}$ is bounded in $W^{1, p}\left(\mathbf{R}^{n}\right)$.

Proof: The claim follows from the proof of Theorem 4.2. In particular, see formula (4.4).

Remarks 4.6. (1) The claim of Theorem 4.2 holds true under the weaker assumption that $\mathcal{B}$ differentiates every $L^{q}\left(\mathbf{R}^{n}\right)$ with $q<p$. To see this we recall that if $\mathcal{B}$ differentiates $L^{q}\left(\mathbf{R}^{n}\right)$ for some $q<p$, then by Theorem 6.4.11 of [dG2] the maximal function related to the basis $\mathcal{B}$ is of weak type $(q, q)$. We can obviously replace the $(1,1)$ weak type estimate in the proof of Theorem 4.2 with the weak type $(q, q)$ estimate.

(2) The condition in Theorem 4.2 is far from being necessary. The basis consisting of all bounded open rectangles with sides parallel to the coordinate axes does not differentiate $L^{1}\left(\mathbf{R}^{n}\right)$ but it differentiates every $L^{q}\left(\mathbf{R}^{n}\right)$ with $1<q<\infty$. Using (1) we may conclude that it also differentiates $W^{1, p}\left(\mathbf{R}^{n}\right)$. 
(3) If $p>n$, then by the Sobolev embedding theorem every function $u \in W^{1, p}\left(\mathbf{R}^{n}\right)$ is Hölder continuous after a possible redefinition on a set of measure zero. Hence every differentiation basis differentiates $W^{1, p}\left(\mathbf{R}^{n}\right)$. In particular, the basis consisting of all rectangles with arbitrary orientations differentiates $W^{1, p}\left(\mathbf{R}^{n}\right)$ but it does not differentiate $L^{q}\left(\mathbf{R}^{n}\right)$ for any $1 \leq q<\infty$.

(4) We construct an example of a differentiation basis which does not differentiate $W^{1, p}\left(\mathbf{R}^{n}\right)$ when $1<p \leq n$. Consider the basis $\mathcal{B}$ obtained by taking the translations of all the sets

$$
((0, a) \times \cdots \times(0, a)) \cup\left(\left(0, a_{1}\right) \times \cdots \times\left(0, a_{n}\right)\right),
$$

where $a>0$ and $a_{i}>0, i=1,2, \ldots, n$. Hence a typical element of $\mathcal{B}$ consists of a cube and an interval attached to it.

Let $1<p<n$ and let

$$
E=\bigcup_{k=1}^{\infty}\left\{q_{k}\right\}
$$

be a countable and dense set in the unit ball $B(0,1)=\left\{x \in \mathbf{R}^{n}:|x|<1\right\}$. Then the function

$$
v(x)=\max (0,1-|x|) \sum_{k=1}^{\infty} 2^{-k}\left|x-q_{k}\right|^{-\alpha}, \quad 0<\alpha<\frac{n-p}{p},
$$

belongs to $W^{1, p}\left(\mathbf{R}^{n}\right)$ but the upper derivative $\bar{D} v(x)=\infty$ for every $x \in B(0,1)$. To see this we observe that if $x \in E$, then the cubes $Q(x, r)$ with the center $x$ and the side length $r$ belong to $\mathcal{B}(x)$ and

$$
\limsup _{r \rightarrow 0} f_{Q(x, r)} v(y) d y=\infty .
$$

On the other hand, if $x \in B(0,1) \backslash E$, then for each $r>0$ there is a set $B \in \mathcal{B}(x)$ such that $\operatorname{diam}(B)<r$ and

$$
f_{B} v(y) d y>\frac{1}{r}
$$

The set $B$ can be constructed by using the density of $E$ and (4.7).

If $p=n$, then

$$
v(x)=\max (0,1-|x|) \sum_{k=1}^{\infty} 2^{-k} \log \log \left(1+\frac{1}{\left|x-q_{k}\right|}\right),
$$

belongs to $W^{1, n}\left(\mathbf{R}^{n}\right)$ and $\bar{D} v(x)=\infty$ for every $x \in B(0,1)$.

By Theorem 2.11 the basis $\mathcal{B}$ does not differentiate $W^{1, p}\left(\mathbf{R}^{n}\right)$. Observe, that $\mathcal{B}$ does not differentiate any $L^{q}\left(\mathbf{R}^{n}\right)$ with $1 \leq q<\infty$. 


\section{References}

[C] J. CheEger, Differentiability of Lipschitz functions on metric measure spaces, Geom. Funct. Anal. 9(3) (1999), 428-517.

[EG] L. C. Evans And R. F. Gariepy, "Measure theory and fine properties of functions", Studies in Advanced Mathematics, CRC Press, Boca Raton, FL, 1992.

[FKS] E. B. Fabes, C. E. Kenig and R. P. Serapioni, The local regularity of solutions of degenerate elliptic equations, Comm. Partial Differential Equations 7(1) (1982), 77-116.

[GT] D. Gilbarg and N. S. Trudinger, "Elliptic partial differential equations of second order", Second edition, Grundlehren der Mathematischen Wissenschaften 224, Springer-Verlag, Berlin, 1983.

[dG1] M. DE GuZMÁN, "Differentiation of integrals in $\mathbf{R}^{n}$ ", Lecture Notes in Mathematics 481, Springer-Verlag, Berlin-New York, 1975 .

[dG2] M. DE GuZmán, "Real variable methods in Fourier analysis", North-Holland Mathematics Studies 46, Notas de Matemática 75, North-Holland Publishing Co., Amsterdam-New York, 1981.

[Ha] P. HaJŁasz, Sobolev spaces on an arbitrary metric space, Potential Anal. 5(4) (1996), 403-415.

[HaKo1] P. HajŁasz and P. Koskela, Sobolev meets Poincaré, $C$. $R$. Acad. Sci. Paris Sér. I Math. 320(10) (1995), 1211-1215.

[HaKo2] P. HajŁasz and P. Koskela, Sobolev met Poincaré, Mem. Amer. Math. Soc. 145(688) (2000), 101 pp.

[HKM] J. Heinonen, T. Kilpeläinen and O. Martio, "Nonlinear potential theory of degenerate elliptic equations", Oxford Mathematical Monographs. Oxford Science Publications, The Clarendon Press, Oxford University Press, New York, 1993.

[HeKo] J. Heinonen and P. Koskela, Quasiconformal maps in metric spaces with controlled geometry, Acta Math. 181(1) (1998), $1-61$.

[Kil] T. KilpeläInen, A remark on the uniqueness of quasi continuous functions, Ann. Acad. Sci. Fenn. Math. 23(1) (1998), 261-262.

[KKM $]$ T. Kilpeläinen, J. Kinnunen and O. Martio, Sobolev spaces with zero boundary values on metric spaces, Potential Anal. 12(3) (2000), 233-247. 
[Kin] J. Kinnunen, The Hardy-Littlewood maximal function of a Sobolev function, Israel J. Math. 100 (1997), 117-124.

[KL] J. Kinnunen and V. Latvala, Lebesgue points for Sobolev functions on metric spaces, Rev. Mat. Iberoamericana $\mathbf{1 8 ( 3 )}$ (2002), 685-700.

[KM] J. Kinnunen and O. Martio, The Sobolev capacity on metric spaces, Ann. Acad. Sci. Fenn. Math. 21(2) (1996), 367-382.

[Sh] N. Shanmugalingam, Newtonian spaces: an extension of Sobolev spaces to metric measure spaces, Rev. Mat. Iberoamericana 16(2) (2000), 243-279.

[St] E. M. SteIn, "Harmonic analysis: real-variable methods, orthogonality, and oscillatory integrals", Princeton Mathematical Series 43, Monographs in Harmonic Analysis III, Princeton University Press, Princeton, NJ, 1993.

[Y] K. Yosida, "Functional analysis", Sixth edition, Grundlehren der Mathematischen Wissenschaften 123, Springer-Verlag, Berlin-New York, 1980.

Petteri Harjulehto:

Department of Mathematics and Statistics

P. O. Box 68 (Gustaf Hällströmin katu 2b)

FIN-00014 University of Helsinki

Finland

E-mail address: petteri.harjulehto@helsinki.fi

Juha Kinnunen:

Department of Mathematical Sciences

P. O. Box 3000

FIN-90014 University of Oulu

Finland

E-mail address: juha.kinnunen@oulu.fi

Primera versió rebuda el 22 d'octubre de 2003, darrera versió rebuda el 16 de juny de 2004. 
